\title{
BMJ Open Identifying the risk factors for catheter- associated urinary tract infections: a large cross-sectional study of six hospitals
}

\author{
Allison S Letica-Kriegel, ${ }^{1}$ Hojjat Salmasian, ${ }^{2,3}$ David K Vawdrey, ${ }^{4,5}$ \\ Brett E Youngerman, ${ }^{6}$ Robert A Green, ${ }^{7,8}$ E Yoko Furuya, ${ }^{9,10}$ David P Calfee, ${ }^{10,11}$ \\ Rimma Perotte ${ }^{\oplus, 12}$
}

To cite: Letica-Kriegel AS, Salmasian H, Vawdrey DK, et al. Identifying the risk factors for catheter-associated urinary tract infections: a large cross-sectional study of six hospitals. BMJ Open 2019;9:e022137. doi:10.1136/ bmjopen-2018-022137

- Prepublication history and additional material for this paper are available online. To view these files, please visit the journal online (http://dx.doi. org/10.1136/bmjopen-2018022137).

Received 5 February 2018 Revised 17 December 2018 Accepted 8 January 2019

Check for updates

(C) Author(s) (or their employer(s)) 2019. Re-use permitted under CC BY-NC. No commercial re-use. See rights and permissions. Published by BMJ.

For numbered affiliations see end of article.

Correspondence to Dr Rimma Perotte; rimmap@gmail.com

\section{ABSTRACT}

Motivation Catheter-associated urinary tract infections (CAUTI) are a common and serious healthcare-associated infection. Despite many efforts to reduce the occurrence of CAUTI, there remains a gap in the literature about CAUTI risk factors, especially pertaining to the effect of catheter dwell-time on CAUTI development and patient comorbidities.

Objective To examine how the risk for CAUTI changes over time. Additionally, to assess whether time from catheter insertion to CAUTI event varied according to risk factors such as age, sex, patient type (surgical vs medical) and comorbidities.

Design Retrospective cohort study of all patients who were catheterised from 2012 to 2016, including those who did and did not develop CAUTIs. Both paediatric and adult patients were included. Indwelling urinary catheterisation is the exposure variable. The variable is interval, as all participants were exposed but for different lengths of time. Setting Urban academic health system of over 2500 beds. The system encompasses two large academic medical centres, two community hospitals and a paediatric hospital.

Results The study population was 47926 patients who had 61047 catheterisations, of which 861 (1.41\%) resulted in a CAUTI. CAUTI rates were found to increase non-linearly for each additional day of catheterisation; CAUTI-free survival was $97.3 \%$ (Cl: 97.1 to 97.6 ) at 10 days, $88.2 \%$ (Cl: 86.9 to 89.5 ) at 30 days and $71.8 \%$ (Cl: 66.3 to 77.8 ) at 60 days. This translated to an instantaneous HR of. $49 \%-1.65 \%$ in the $10-60$ day time range. Paraplegia, cerebrovascular disease and female sex were found to statistically increase the chances of a CAUTI. Conclusions Using a very large data set, we demonstrated the incremental risk of CAUTI associated with each additional day of catheterisation, as well as the risk factors that increase the hazard for CAUTI. Special attention should be given to patients carrying these risk factors, for example, females or those with mobility issues.

\section{INTRODUCTION}

Catheter-associated urinary tract infections (CAUTI) continue to be among the most
Strengths and limitations of this study

- The analysis focused on a very large population of patients: 4 years of data across 61000 catheterisations and over 47000 patients.

- The methodology not only controls for age, sex and patient type but patient comorbidities as well.

- The definition used for CAUTI was changed by the Centers for Disease Control and Prevention in January 2015 and therefore the CAUTI population in our study is not homogeneous.

- The capabilities of the electronic health record limited our ability to collect some granular data that may have supplemented the analysis.

common healthcare-associated infections in the USA. In 2011, there were an estimated 93000 cases of CAUTI in US acute care hospitals. ${ }^{1}$ CAUTIs can lead to more serious complications such as sepsis and endocarditis, and it is estimated that over 13000 deaths each year are associated with healthcare-associated UTIs. ${ }^{2}$

For an infection to be classified as a CAUTI under guidelines published by the US Centers for Disease Control and Prevention (CDC), a patient must have: (a) had an indwelling urinary catheter for more than 2 days by the date of event (with 'day one' being the day of catheter insertion); (b) one sign or symptom including fever, suprapubic tenderness, costovertebral angle tenderness, urinary frequency or urgency or dysuria; and (c) urine culture with more than $10^{5} \mathrm{CFU} /$ $\mathrm{mL}$ of one bacterial species (non-bacterial pathogens have been excluded since 2015). As it is estimated that $69 \%$ of CAUTI events are avoidable, ${ }^{3}$ the US Department of Health and Human Services spearheaded national efforts in 2009 to reduce CAUTI rate. ${ }^{4}$ The efforts undertaken to reduce CAUTI rates 
include avoiding unnecessary catheterisation, reducing duration of catheterisation (eg, by using reminder systems to encourage catheter removal when the catheter is no longer indicated), emphasising antiseptic technique for insertion and using hydrophilic-coated catheters. ${ }^{56}$

Despite advances in prevention guidelines, there remains a lack of knowledge concerning the risk factors for CAUTI. A seminal study from Garibaldi and colleagues in 1974 examined 405 patients with indwelling urinary catheters, concluding that female sex, age greater than 50 years, higher severity of illness, non-surgical illness and no systemic antibiotics were independent risk factors for the development of CAUTI (defined then as bacteriuria of more than $10^{2} \mathrm{CFU} / \mathrm{mL}$ ). ${ }^{7}$ Importantly, Garibaldi also reported that catheter dwell-time (ie, number of days spent catheterised) was a significant risk factor for CAUTI, with a $7.4 \%$ risk of infection in the 24 hours following insertion, and a steady $8.1 \%$ risk increase each subsequent day for the first 7 days. ${ }^{7}$ Further studies in the 1980s reproduced these risk factors, adding catheter care violations and non-sealed catheter junctions as risk factors as well. ${ }^{8}$

Overall, there have been very few studies that focus on understanding CAUTI risk factors, including dwelltime of the catheter. ${ }^{9}{ }^{10}$ To the best of our knowledge, no study since 1974 has attempted to provide an estimate of the daily risk of maintaining a urinary catheter, a principle that could have significant implications in refining CAUTI prevention guidelines. Further, additional risk factors such as comorbidities need to be better identified. We conducted a retrospective review of data from electronic health records to help illuminate CAUTI risk factors. ${ }^{11}$

The objective of this study was to examine how the risk for CAUTI changes over time and identify the risk factors for CAUTI by analysing data from a large electronic health record (EHR) dataset of routine nursing documentation.

\section{METHODS \\ Setting}

The study was conducted at an urban academic health system comprising two large academic medical centres, two community hospitals and a paediatric hospital. Together the hospitals had over 2500 beds. The Columbia University Irving Medical Center's Institutional Review Board reviewed the study and granted approval.

\section{Patient and public involvement}

This research was conducted in reaction to the efforts focused on reducing CAUTI rates, which is a concern to both providers and patients alike. However, the study is a retrospective analysis of data collected as part of care; therefore, there was no direct involvement of patients in design, recruitment and conduct of the study.
Review of electronic health record nursing documentation

We reviewed all infections associated with catheters that occurred between 1 January 2012 and 31 March 2016. For all patients hospitalised at the study sites during this time period, we identified 'catheter days', that is, the number of calendar days during which the patient had an indwelling urinary catheter (IUC) as recorded in nursing flowsheet documentation in the EHR. The presence of CAUTI was extracted from Infection Prevention and Control logs as they are manually assigned after detailed review, using the definition that is set forth by National Healthcare Safety Network within the CDC. Both flowsheet documentation and CAUTI presence logs contained patient medical record number and admission date, these two identifiers were used to link the IUC data with the CAUTI events. If multiple CAUTI events occurred during one IUC period, only the earliest CAUTI was included in our analysis (ie, our analysis examined event-free survival for each IUC period). However, if there was a reinsertion, this was considered a new IUC period and therefore a CAUTI during this new period would be eligible for the study. All ages and all types of infections (bacterial and fungal) were included in the study.

\section{Statistical analysis}

We calculate and report a CAUTI rate per 1000 catheter days using the full population of catheterisations, as is standard. For the subsequent analyses, the population is filtered to remove all catheterisations with an IUC duration of less than 3 days (this is to match the CDC criteria for defining CAUTIs). ${ }^{12}$ To address the issue of missing data, any catheterisations for patients who were missing covariates are removed (online supplementary figure 1).

We performed a time-independent analysis to evaluate differences between catheterisations that resulted in a CAUTI and those that did not. Differences in continuous variables were assessed with a t-test and differences in categorical variables were assessed with a $X^{2}$ test. The time-independent analysis was followed by survival analyses to characterise event-free survival-that is, number of days between IUC insertion and either CAUTI occurrence or IUC removal with no CAUTI event. As CAUTI is defined as infection that occurs any time after the second day of catheter placement, it is impossible for a CAUTI to exist prior to day 3. Therefore, risk for CAUTI begins at day 3 by definition. In our survival analyses, day 3 is considered the start time.

A Kaplan-Meier estimate was computed to evaluate the instantaneous hazard rates for developing a CAUTI and the time-dependent differences in sub-populations found to be significant in the time-independent analysis. The time-dependent differences in infection-free survival rates are reported in the full population, the population stratified by paediatric patients versus adult patients, and stratified by female patients versus male patients.

Finally, after testing the proportional hazard assumption and finding that it is not violated, we performed a Cox proportional hazard analysis. To address potential 
biases we adjusted for a set of confounders. Univariate Cox models were created to assess the effect of each variable's effect on time-to-infection; overall Charlson Comorbidity Index (CCI) score ${ }^{13}$ the presence or absence of the 17 comorbidities that are included in CCI, sex, age and patient type (medical vs surgical) were examined. CCI was computed for each patient using an automated method described by Salmasian et al. ${ }^{14}$ The CCI assigns weights to the 17 different comorbidities and was originally designed to predict 10 year survival in patients (ie, the higher a patient's CCI, the more likely they are to die). The 17 comorbidities included were myocardial infarction, congestive heart failure, peripheral vascular disease, cerebrovascular disease, dementia, chronic pulmonary disease, connective tissue disease, ulcer disease, mild liver disease, diabetes, diabetes with complications, paraplegia, renal disease, cancer, metastatic cancer, severe liver disease and HIV. The variables found to be significant in the univariate models were included in the multivariate Cox proportional hazard model.

All statistical analyses were carried out using the $\mathrm{R}$ programming language ${ }^{15}$ using the survival and survminer packages.

\section{RESULTS}

\section{Catheter-associated urinary tract infection rates}

From January 2012 to March 2016, there were 148631 catheterisations for 115710 patients. The 148631 catheterisation events for the 2012-2016 time period totalled 540494 catheter days. We identified a CAUTI rate of 1.64 per 1000 catheter-days (95\% CI: 1.63 to 1.65$)$ for the total population. The paediatric population $(0-17$ years of age) had a total of 23531 catheter days and a CAUTI rate of 2.08 (95\% CI: 1.56 to 2.78$)$ per 1000 catheter days. The adult population (18+ years of age) had a total of 517335 catheter days and a CAUTI rate of 1.61 (95\% CI: 1.51 to 1.73) per 1000 catheter days, representing a non-statistically significant difference with the paediatric population.

\section{Time-independent analysis}

To align with CDC CAUTI definitions and remove missing data, all catheterisations with missing data or IUC duration of less than 3 days were removed from the analysis. This filtration resulted in a cohort of 47926 patients with 61047 catheterisations and a total of 381951 catheter days (16254 paediatric catheter days and 365697 adult catheter days). From this total population of patients with IUCs and without missing data, there were 861 CAUTI events $(1.41 \%(1.32 \%$ to $1.51 \%))$. Overall median duration of IUC from first insertion was 7 days $(\mathrm{IQR}=10)$ for patients who developed a CAUTI and 4 days $(\mathrm{IQR}=4)$ for those who did not. Median duration of catheterisation for all IUCs was 4 days $(\mathrm{IQR}=4)$.

There were significant differences identified in terms of age, sex and CCI between the patients who developed a CAUTI and those who did not (table 1). The results indicate that paediatric patients and women are more prone to develop CAUTIs. We also see evidence that older but not elderly patients (ages 25-64) are also prone to develop CAUTIs.

\section{Time-to-event analysis}

The Kaplan-Meier curve for all catheterisations is displayed in figure 1. CAUTI-free survival rate was $97.3 \%$ (CI: $97.1 \%$ to $97.6 \%$ ) at 10 days, $88.2 \%$ (CI:86.9\% to

Table 1 Descriptive data for the full population of catheterisations

\begin{tabular}{|c|c|c|c|}
\hline & CAUTI developed $(n=861)$ & No CAUTI developed $(n=60186)$ & $\begin{array}{l}\text { P value for group } \\
\text { differences }\end{array}$ \\
\hline \multicolumn{4}{|l|}{ Age } \\
\hline Mean (years) & 60.2 (SD 21.4) & 62.6 (SD 21.3) & $0.001^{*}$ \\
\hline $0-17$ & $49(5.6 \%)$ & $2836(4.7 \%)$ & \\
\hline $18-24$ & $14(1.6 \%)$ & $1271(2.1 \%)$ & $0.025 \dagger$ \\
\hline $25-44$ & $115(13.4 \%)$ & $6741(11.2 \%)$ & \\
\hline $45-64$ & $253(29.4 \%)$ & $16410(27.3 \%)$ & \\
\hline $65+$ & $430(49.9 \%)$ & $32928(54.7 \%)$ & \\
\hline \multicolumn{4}{|l|}{ Sex } \\
\hline Male & $335(38.9 \%)$ & $30669(51.0 \%)$ & $<0.001 \uparrow$ \\
\hline \multicolumn{4}{|l|}{ Patient Type } \\
\hline Surgical & $613(71.2 \%)$ & $41988(69.8 \%)$ & $0.383 \dagger$ \\
\hline \multicolumn{4}{|l|}{ Comorbidities } \\
\hline Charlson Comorbidity Score & 3.0 (SD 2.7) & 2.6 (SD 2.6) & $<0.001^{*}$ \\
\hline
\end{tabular}

CAUTI, catheter-associated urinary tract infection.

The table compares descriptive statistics for catheterisations between patients who developed CAUTI and who did not develop it *Student's t-test

$\dagger \mathrm{X}^{2}$ analysis. 

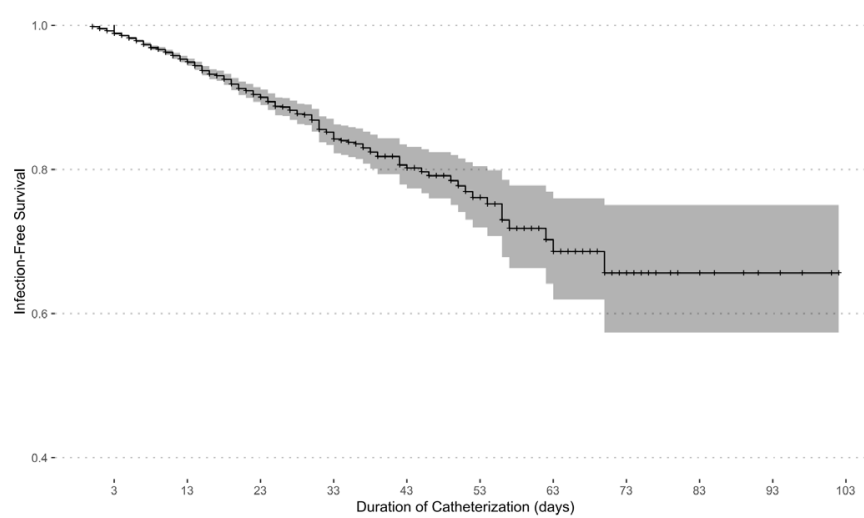

At risk 61,047 1,519

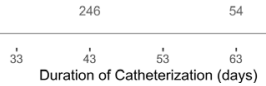

s)

10

Figure 1 Kaplan-Meier survival curve for all catheterisations. The $\mathrm{x}$-axis begins on day 3 as only patients who had catheters inserted for at least two full days can develop a catheter-associated urinary tract infection, according to the Centers for Disease Control and Prevention definition. The number of patients at risk after each 20 day interval is represented in the table below the figure. Minimal survival rate of. 66 is reached on day 73 when 23 patients remain in the study sample.

$89.5 \%$ ) at 30 days and $71.8 \%$ (CI: $66.3 \%$ to $77.8 \%$ ) at 60 days. The instantaneous hazard is depicted in figure 2. From left to right, the number of patients with that many days of catheterisation decreases, and hence the CI for the smoothed model widens. The B-spline smoothing model estimates that instantaneous hazard to be the highest at around 40 days.

Analysing disease-free survival for different patient cohorts using a Kaplan-Meier estimate demonstrated that paediatric patients were at a higher risk of developing a CAUTI (log rank $\mathrm{p}=0.007$ ) (figure 3A). The KaplanMeier infection-free probability estimates at 30 days were 0.88 (0.87 to 0.90 ) for adult and 0.87 (0.82 to 0.93 ) for

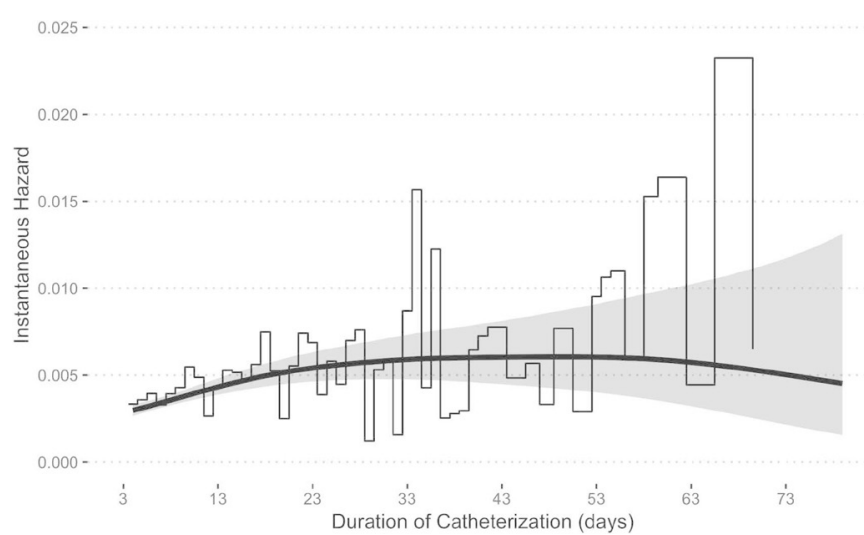

Figure 2 Instantaneous hazard curve. The instantaneous hazard is derived using the Kaplan-Meier estimates. The B-spline smoothing model estimates that instantaneous hazard to be the highest at around 40 days. The graph is truncated after the last catheter-associated urinary tract infection event occurs. paediatric patients with the largest difference in survival occurring between days 30 and 55. Paediatric girls were found to be over three times as likely to develop a CAUTI than paediatric boys. Additionally, women were found to be more likely to develop CAUTI than men (log-rank $\mathrm{p}<0.0001$ ) (figure 3B). The Kaplan-Meier infection-free probability estimates at 30 days were 0.84 (0.82 to 0.87 ) for women and 0.92 (0.90 to 0.93) for men patients with the largest difference in survival occurring between days 10 and 60.

Cox proportional hazards models identified additional risk factors for CAUTI. The univariate Cox proportional hazards models found the following variables as significantly affecting time-to-infection: age, sex, CCI, myocardial infarction, congestive heart failure, cerebrovascular disease, pulmonary disease, connective tissue disease, paraplegia, renal disease and severe liver disease. The multivariate Cox model reduced the set of significant variables: congestive heart failure was associated with a HR 0.75 (064 to 0.89) $(\mathrm{p}<0.001)$, whereas cerebrovascular disease and paraplegia increased the risk by 1.78 (1.53 to 2.08) $(\mathrm{p}<0.001)$ and 1.40 (1.11 to 1.77$)(\mathrm{p}=0.005)$, respectively (figure 4 ). The hazard for males was found to be $0.56(0.48$ to 0.64$))$.

\section{DISCUSSION}

CAUTIs pose a significant burden on patients, both in terms of morbidity and mortality. Apart from the clear harm posed to patients, governmental pressure has helped hospitals across the country focus even more effort on CAUTI reduction. CAUTI rates are incorporated into both government quality ratings through the Centers for Medicaid and Medicare quality star ratings, as well as financial penalties through the Hospital Acquired Condition Reduction Penalty programme.

However, while some CAUTI reduction efforts have shown positive results, ${ }^{3} 16$ CAUTI rates in the USA still increased by $6 \%$ from 2009 to $2013 .{ }^{3}$ CAUTIs remain one of the most common nosocomial infections; however, there is little evidence on what specific factors lead to these infections. If we are able to identify factors that are associated with the development of CAUTIs, we will then be able to modulate our preventive strategy accordingly, specifically modifying the standard of care for hospital practice as well as implementing targeted electronic alerts, such as alerting at a higher frequency for paediatric patients.

Prompt removal of IUC at the earliest possibility has been a cornerstone of CAUTI reduction programmes in the published literature, and the toolkit developed by the Agency for Healthcare Research and Quality to reduce CAUTIs also emphasises on prompt removal of IUCs. ${ }^{17}$ Nevertheless, evidence on the additive hazard of IUC duration in development of CAUTI had not been updated for more than three decades. Our study is the first to revisit this association for a very large cohort of 
A

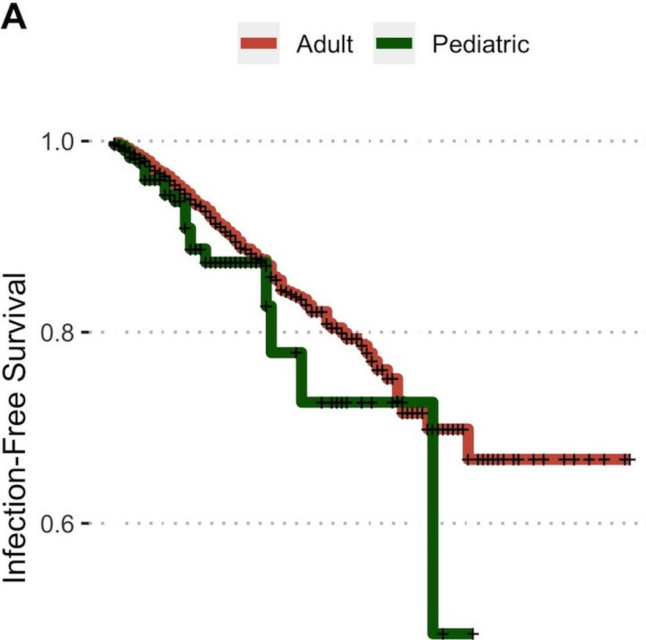

$0.4-$

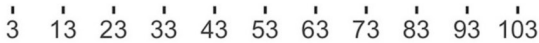
Duration of Catheterization (days)
B

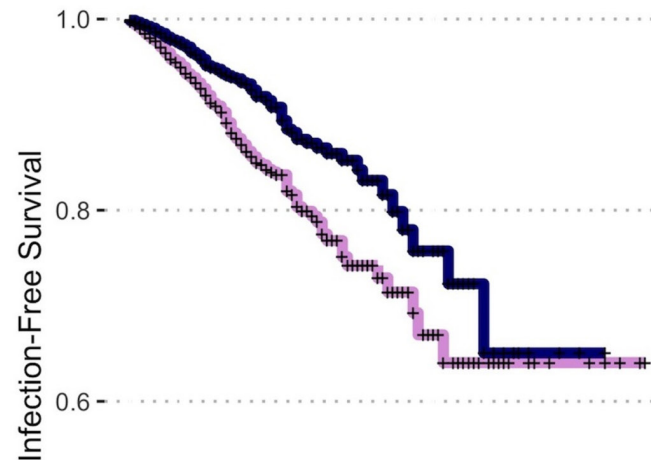

$$
\begin{aligned}
& 0.4-
\end{aligned}
$$

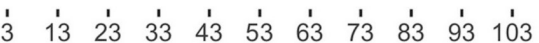

$$
\begin{aligned}
& \text { Duration of Catheterization (days) }
\end{aligned}
$$

Figure 3 Infection-free survival stratified by sex and age group. (A) Kaplan-Meier survival curve comparing adult (18+ years old) versus paediatric (0-17 years old) groups (log-rank $p=0.007)$. (B) Kaplan-Meier survival curve comparing men and women (logrank $\mathrm{p}<0.0001)$.

patients and provide novel evidence about the various risk factors for CAUTI in hospitalised patients.

\section{Root cause analysis for CAUTI events}

In addition to the EHR-derived IUC data, we were able to obtain the records from a collection of root cause analysis (RCA) performed for patients who contracted a CAUTI. RCAs are routinely performed at healthcare institutions and have been previously found to help in understanding the underlying causes for infection. ${ }^{18}$ Our study had RCA data available for $10 \%$ of the total CAUTIs in the study population. Analysis of the RCA data identified that the most common indications for catheterisation were recording inputs and outputs, critical illness, peri-operative status and diuresis. The most common contributing factors for CAUTI that were identified by the clinical teams and infection preventionists were comorbidities, lapses in catheter care protocols, active infection, faecal incontinence and duration of IUC. The duration of IUC was identified as a contributing factor for $16.5 \%$ of the CAUTI cases, however, for almost $25 \%$ of the cases reviewed in the RCAs, the clinical teams and infection preventionists stated that the catheters could have been removed earlier.

\section{Contributions to the CAUTI risk factor literature}

Our study upholds previous findings and provides new insights as well. As has been previously reported, longer

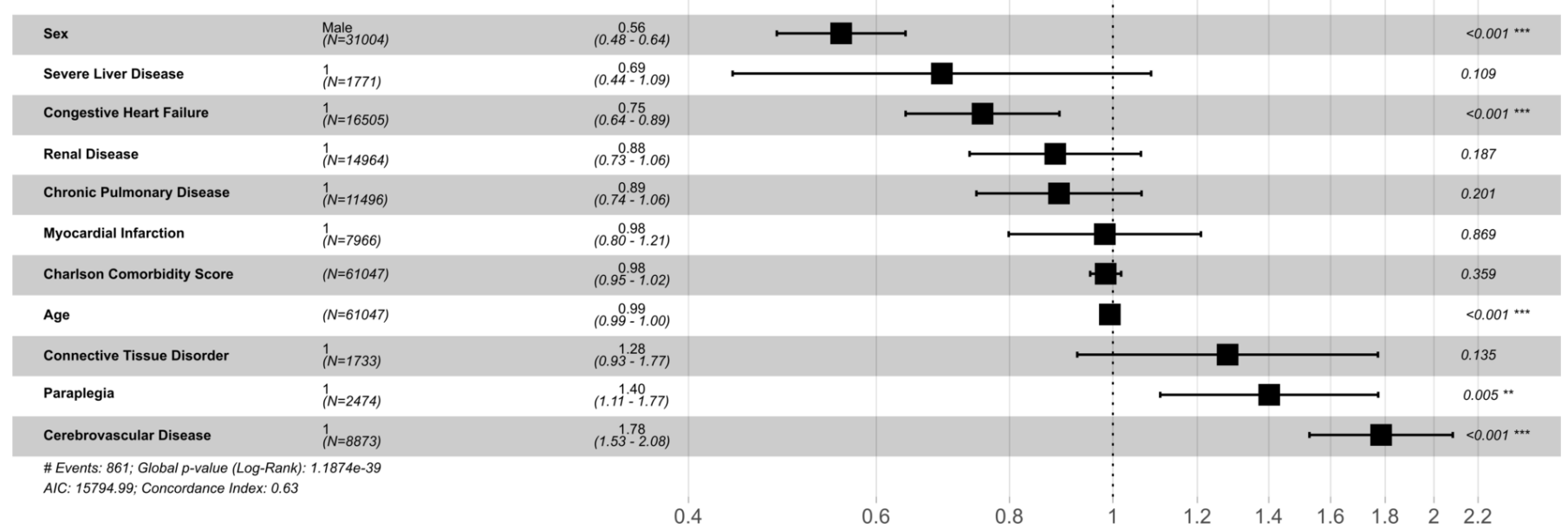

Figure 4 Hazard ratios derived from the Cox proportional hazards model. The presence of a comorbidity is represented by a ' 1 ', that is, 1771 patients had a severe liver disease diagnosis. Only comorbidities that were found to be significant in a univariate analysis are included in the multivariate model and figure. 
duration of catheterisation and female sex are both risk factors for the development of CAUTI. ${ }^{79-22}$ Interestingly, using the same adult age brackets (18-50 years old and older than 50 years) as the Garibaldi et al 1974 study, we found that younger adults had a 1.3 (95\% CI: 1.1 to 1.5$)$ times greater hazard for developing a CAUTI $(p=0.007)$, which is the opposite of the results in the original study. When we adjust for life expectancy differences and look at patient ages $18-64$ vs patient ages $65+$, the risk of CAUTI remained higher in younger patients than in older patients. However, when adding comorbidities to the analysis, age was no longer found to be a significant factor, implying that age in itself may not be a risk factor.

As the data for CAUTIs in paediatric patients is limited, the current study supplements available medical literature. Goudie $e t a l^{23}$ studied healthcare-associated infections in the paediatric population and found that the risk of CAUTIs in girls was more than three times higher than that in boys. Our study found a very similar significant difference between the sexes. In addition, our study showed that paediatric patients had a higher risk of being infected than their adult counterparts, suggesting that even more caution should be undertaken in children with catheters.

While there is little literature to compare to our findings regarding the CCI, there were both expected and unexpected findings with this data. Both stroke (cerebrovascular disease) and paraplegia being risk factors for CAUTI is understandable, as neurologic units have been shown to have higher rates of CAUTI as compared with other units. ${ }^{24}$ As was demonstrated by Titsworth et $a l^{25}$ introducing UTI bundles and practicing a higher-level vigilance in neurological intensive care units can decrease the rate of infection in this subset of patients. Although the CCI was created to predict mortality and not infection susceptibility, it was unexpected that the overall comorbidity score did not affect the risk of CAUTI as we expect increased comorbidity burden to increase risk of infection. The most surprising result was congestive heart failure (CHF) was associated with a lower risk of CAUTI in our population. One possible explanation for this result is that many patients with CHF have a very specific indication for catheterisation, namely diuresis, and are monitored vigilantly for euvolemic fluid status, which prompts timely removal of the catheter. The current analysis does not distinguish between patients with a long-standing diagnosis of $\mathrm{CHF}$ and patients who are in the hospital for a CHF exacerbation, so this data cannot be extrapolated to infer that patients with CHF exacerbation have a lower risk of CAUTI, but this is an area that is important for future analysis.

While previous studies have reported on various metrics of CAUTI risk relative to IUC duration, our study demonstrates a non-linear increase in the cumulative risk hazard as duration increases, suggesting that each extra day of catheterisation incrementally increases the risk of CAUTI. As the RCA data revealed that almost one quarter of patients could have had their catheters removed earlier and the EHR-derived data identified the risk for CAUTI increases non-linearly each day, these results can help encourage automated alerts for the removal of IUCs and inform their timing.

Our study also has a number of limitations. First, the definition used for CAUTI was changed by the CDC in January 2015 and therefore the CAUTI population in our study is not homogeneous; for instance, non-bacterial forms of UTI could be counted as CAUTI in the data from 2012 to 2014, but not in the data from 2015 and $2016 .{ }^{26}$ If we assume that the daily additional risk of CAUTI differs in fungal versus bacterial UTI, then this heterogeneity in the data may have confounded our results. Second, the capabilities of the EHR limited our ability to collect some granular data that may have supplemented the analysis. Lastly, some patients had more than one CAUTI catheterisation events in our data. While typically these catheterisations occurred in different admissions, it is possible that patient-level factors may contribute to the risk of infection, that is, not all of our data points are completely independent. We tried to model these patient-level factors using the covariates in the adjusted model, but this model may not have sufficiently addressed the issue of dependent data points. Despite these limitations, we do believe our study is generalisable as it includes a large, heterogeneous subset of patients at a tertiary care centre in a large metropolitan area.

\section{CONCLUSIONS}

Using a very large EHR-derived dataset, our findings indicate that IUC dwell time is a significant risk factor for patients who develop CAUTIs, even when controlling for sex, age and patient comorbidities. We also identify female sex, paediatric age and neurological issues as risk factors for CAUTIs. Our study finds that approximately $12 \%$ of patients who have a catheter inserted for 30 days will develop a CAUTI. The findings of this study can help guide efforts for future CAUTI reduction programmes.

\section{Author affiliations}

${ }^{1}$ Department of Surgery, Massachusetts General Hospital, Boston, Massachusetts, USA

2Department of Quality and Safety, Brigham and Women's Hospital, Boston, Massachusetts, USA

${ }^{3}$ Division of General Internal Medicine, Harvard Medical School, New York, USA

${ }^{4}$ Value Institute, NewYork-Presbyterian Hospital, New York, USA

${ }^{5}$ Department of Biomedical Informatics, Columbia University Irving Medical Center, New York, USA

${ }^{6}$ Department of Neurological Surgery, Columbia University Irving Medical Center, New York, USA

${ }^{7}$ Department of Quality and Patient Safety, NewYork-Presbyterian Hospital, New York, USA

${ }^{8}$ Department of Emergency Medicine, Columbia University Irving Medical Center, New York, USA

${ }^{9}$ Department of Medicine, Columbia University Irving Medical Center, New York, USA ${ }^{10}$ Department of Infection Prevention and Control, NewYork-Presbyterian Hospital, New York, USA

${ }^{11}$ Department of Medicine, Weill Cornell Medicine, New York, USA

${ }^{12}$ Department of Biomedical Informatics, Columbia University Irving Medical Center, New York, USA 
Acknowledgements We are grateful to the reviewers of this manuscript for helping us greatly improve the manuscript through the peer-review process.

Contributors Concept and design: ASL-K, RP, HS, DKV. Acquisition, analysis or interpretation of data: ASL-K, RP, HS, BEY. Drafting of the manuscript: DPC, EYF, RAG, ASL-K, RP, HS, DKV, BEY. Critical revision of the manuscript for important intellectual content: DPC, EYF, RAG, ASL-K, RP, HS, DKV, BEY. Statistical analysis: ASL-K, RP, HS. Supervision: RP, HS, DKV.

Funding The authors have not declared a specific grant for this research from any funding agency in the public, commercial or not-for-profit sectors.

Competing interests None declared.

Patient consent for publication Not required.

Provenance and peer review Not commissioned; externally peer reviewed.

Data sharing statement No additional data available.

Open access This is an open access article distributed in accordance with the Creative Commons Attribution Non Commercial (CC BY-NC 4.0) license, which permits others to distribute, remix, adapt, build upon this work non-commercially, and license their derivative works on different terms, provided the original work is properly cited, appropriate credit is given, any changes made indicated, and the use is non-commercial. See: http://creativecommons.org/licenses/by-nc/4.0/.

\section{REFERENCES}

1. Magill SS, Edwards JR, Bamberg W, et al. Multistate pointprevalence survey of health care-associated infections. N Engl J Med Overseas Ed 2014;370:1198-208.

2. Klevens RM, Edwards JR, Richards CL, et al. Estimating health care-associated infections and deaths in U.S. hospitals, 2002. Public Health Rep 2007;122:160-6.

3. Saint S, Greene MT, Krein SL, et al. A program to prevent catheterassociated urinary tract infection in acute care. $N$ Engl J Med 2016;374:2111-9.

4. Department of Health and Human Services. National action plan to prevent health care-associated infections: road map to elimination. Washington, DC: Department of Health and Human Services, 2009.

5. Lo E, Nicolle LE, Coffin SE, et al. Strategies to prevent catheterassociated urinary tract infections in acute care hospitals: 2014 update. Infection Control \& Hospital Epidemiology 2014;35:S32-47.

6. Gould CV, Umscheid CA, Agarwal RK, et al. Guideline for prevention of catheter-associated urinary tract infections. 2009 https://www. cdc.gov/infectioncontrol/guidelines/cauti/

7. Garibaldi RA, Burke JP, Dickman ML, et al. Factors predisposing to bacteriuria during indwelling urethral catheterization. N Engl J Med 1974;291:215-9.

8. Stamm WE. Catheter-associated urinary tract infections: epidemiology, pathogenesis, and prevention. Am J Med 1991;91:S65-71.
9. Al-Hazmi H. Role of duration of catheterization and length of hospital stay on the rate of catheter-related hospital-acquired urinary tract infections. Res Rep Urol 2015;7:41-7.

10. Karp NE, Kobernick EK, Kamdar NS, et al. 36: Length of catheter use after hysterectomy as a risk factor for UTI. Am J Obstet Gynecol 2016;214:S485-6.

11. Michelson JD, Pariseau JS, Paganelli WC. Assessing surgical site infection risk factors using electronic medical records and text mining. Am J Infect Control 2014;42:333-6.

12. CDC. Urinary Tract Infection (Catheter-Associated Urinary Tract Infection [CAUTI] and Non-Catheter-Associated Urinary Tract Infection [UTI] and other Urinary System Infection [USI]) Events. 2017 http://www.cdc.gov/nhsn/pdfs/pscmanual/7psccauticurrent.pdf

13. Charlson ME, Pompei $P$, Ales KL, et al. A new method of classifying prognostic comorbidity in longitudinal studies: development and validation. J Chronic Dis 1987;40:373-83.

14. Salmasian H, Freedberg DE, Friedman C. Deriving comorbidities from medical records using natural language processing. J Am Med Inform Assoc 2013;20:e239-42.

15. Core Team R. R: A language and environment for statistical computing. R Foundation for Statistical Computing. Vienna, Austria, 2013.

16. Taha H, Raji SJ, Khallaf A, et al. Improving catheter associated urinary tract infection rates in the medical units. BMJ Qual Improv Rep 2017;6:u209593.w7966.

17. Agency for Healthcare Research and Quality. Toolkit for reducing catheter-associated urinary tract infections in hospital units: implementation guide: AHRQ Publication, 2015:15.

18. Venier AG. Root cause analysis to support infection control in healthcare premises. J Hosp Infect 2015;89:331-4.

19. Kunin CM, McCormack RC. Prevention of catheter-induced urinary-tract infections by sterile closed drainage. N Engl J Med 1966;274:1155-61.

20. Loeb M, Hunt D, O'Halloran K, et al. Stop orders to reduce inappropriate urinary catheterization in hospitalized patients: a randomized controlled trial. J Gen Intern Med 2008;23:816-20.

21. Tambyah PA, Maki DG. Catheter-associated urinary tract infection is rarely symptomatic: a prospective study of 1,497 catheterized patients. Arch Intern Med 2000;160:678-82.

22. Vincitorio D, Barbadoro P, Pennacchietti L, et al. Risk factors for catheter-associated urinary tract infection in Italian elderly. Am J Infect Control 2014;42:898-901.

23. Goudie A, Dynan L, Brady PW, et al. Costs of venous thromboembolism, catheter-associated urinary tract infection, and pressure ulcer. Pediatrics 2015;136:432-9.

24. Dudeck MA, Edwards JR, Allen-Bridson K, et al. National Healthcare Safety Network report, data summary for 2013, device-associated module. Am J Infect Control 2015;43:206-21.

25. Titsworth WL, Hester J, Correia T, et al. Reduction of catheterassociated urinary tract infections among patients in a neurological intensive care unit: a single institution's success. J Neurosurg 2012;116:911-20.

26. Centers for Disease Control and Prevention. FAQs: NHSN CAUTI Definition \& Rebaseline. 2015 https://www.cdc.gov/nhsn/pdfs/ rebaseline/faq-cauti-rebaseline.pdf 